\title{
A NEWTON-RAPHSON VERSION OF THE MULTIVARIATE DYNAMIC ROBBINS-MONRO PROCEDURE
}

\author{
El Sayed Sorour \\ Military Technical College, \\ Department of Mathematics.
}

\section{ABSTRACT}

Let $M$ be a function from $R^{k}$ to $R^{k}$, let $\theta_{n}, n=1,2, \ldots$ be (unknown) vector numbers, the first $\theta_{1}$ being the unique root of the equation $M(x)=0$, set $M_{1}(x)=M(x)$, for $n=1,2, \ldots$ set $M_{n}(x)=M\left(x-\theta_{n}-\theta_{1}\right)$ so that $\theta_{n}$ is the unique root of $M_{n}(x)=0$. Initially $M_{n}(x)$ is unknown, but for any $x$ in $R^{k}$ we can observe a random vector $\mathrm{Y}_{\mathrm{n}}(\mathrm{x})$ with conditional expectation $M_{n+1}(x)$. The unknown $\theta_{n}$ can be estimated recursively by the author (1978), that procedure requires the rather restrictive assumption that the infimum of the inner product $\langle x-\theta, M(x)\rangle$ over any compact set not containing $\theta$ be positive, i.e. along each line through $\theta_{1}, M(x)$ is unimodal with minimum $\theta_{1}$. Unlike our previous method, the procedure introduced in this paper does not necessarily attempt to move in the direction of $\theta_{n}$ but except of that random fluctuations it moves in the direction which decreases $\left\|M_{n}(x)\right\|^{2}$, consequently it does not require that $\left\langle x-\theta_{n}, M_{n}(x)\right\rangle$ have a constant signum. This new procedure is a stochastic analog of the Newton-Raphson technique. 


\section{INTRODUCTION}

This paper is concerned with the multivariate dynamic version of the Robbins-Monto [3] stochastic approximation procedure. This problem first studied by Dupac [1]; where the loot of the regression function moves in a specified maner. He discussed in his papers [1]; [2] only the cases where the movement of the root (one-dimensional) or the maximum (multidimensional) can be expressed by a certain linear function of its present location, and where the trend is deterministic. Uosaki, K. [9] discussed some generalization of Dupac's work in the one-dimensional case where the movement of the root can be expressed by a specified non-linear function of its present location. Uosaki's result has been generalized to the multidimensional case by the author (1978). This version begins with an initial costimate $x_{\text {? }}$. Given $x_{2}, x_{2}, \ldots, x_{n}$ one observes $Y_{n}$, such that $E_{n}\left(Y_{n}\right)=M_{n+1}\left(*_{n}^{*}\right)$, where $E_{n}$ denotes the conditional expectation given $x_{1}, x_{2}, \ldots, x_{n}$ and $\stackrel{*}{x}_{n_{1}}=g_{n}\left(x_{n}\right)$ for some function $g_{n}$ from $R^{k} \rightarrow R^{k}$. Then $x_{n+1}$ is defined by

$$
\mathrm{x}_{\mathrm{n}+1}=\stackrel{*}{\mathrm{x}}_{\mathrm{n}}-\mathrm{a}_{\mathrm{n}} \mathrm{Y}_{\mathrm{n}} \ldots \ldots
$$

where $\left\{a_{n}\right\}$ is a suitably choosen positive sequence converging to 0 as $n \longrightarrow \infty$. Let $\theta_{n}$ be the unique root of $M_{n}(x)$. Then $x_{n}-\theta_{n} \rightarrow 0$, under the assumption for every $\varepsilon>0$

$$
\left.\inf _{n \in N} \inf _{n-\theta_{n} \|>\varepsilon} \frac{\left\langle x-\theta_{n}, M_{n}(x)\right\rangle}{\left\|x-\theta_{n}\right\|}\right\rangle 0 .
$$

In fact it can be proved that (1.2) can be replaced by a weaker one, for every $\varepsilon>0$ 


$$
\inf _{n \in N} \underset{\varepsilon \in\left\|x-\theta_{n}\right\|<\varepsilon}{\inf }\left\langle x-\theta_{n}, M_{n}(x)\right\rangle>0
$$

The importance of $(1.2)^{\prime}$ can be easily seen as follows. Suppose that $\operatorname{Sup}\left\{\operatorname{Var} Y: x \in \mathrm{R}^{\mathrm{k}}\right\}<\infty$. Thus from (1.1) we have

$$
\begin{aligned}
& E_{n}\left(\left\|x_{n+1}-\theta_{n+1}\right\|^{2}\right) \leq\left\|x_{n}-\theta_{n}\right\|^{2}\left(1+\mu_{n}\right) \\
& -2 a_{n}(1+o(1))<\stackrel{*}{x}_{n}-\theta_{n+1}, M_{n+1}\left(\stackrel{\star}{x}_{n}\right)>+v_{n}
\end{aligned}
$$

where $\Sigma \mu_{\mathrm{n}}<\infty ; \Sigma \mathrm{v}_{\mathrm{n}}<\infty$. Using (1.2)' and theorem 1 of Robbins and sigmund [4] $\left\|x_{n}-\theta_{n}\right\|$ converges to 0 . Unfortunately, (1.2)' is a rather restrictive assumption, emplying that for each $x$, $M_{n}(x)$ points a-way from $\theta_{n}$. There are many practical examples for functions do not astisfy the condition (1.2)'. An alternative procedure would to apply the multivariate Kiefer-Wolfowitz (KW) to minize $\left\|M_{n}(x)\right\|^{2}$ a fact used by Ruppert (1985) for the ordinary (PM) procedure. We assume that $E_{n}\left(\left\|Y_{n}\right\|^{2}\right)=\left\|M_{n}(x)\right\|^{2}+$ const. Thus the (KW) procedure does not attempt to move towards $\theta_{n}$; but in a direction of decreasing $\left\|M_{n}(x)\right\|^{2}$. If $\theta_{n}$ is the only local minimum of $M_{n}(x)$, we prove that $x_{n}-\theta_{n} \rightarrow 0$ under mild conditions.

\section{NOTATIONS AND ASSUMPTIONS}

2.1 : Let $\mathrm{R}^{\mathrm{k}}$ be the $\mathrm{k}$-dimensional vector space. For $\mathrm{x}$ in $\mathrm{R}^{\mathrm{k}}$ let $x^{i}$ be the ith component of $x$. For $x, y$ in $R^{k}$ we define

$$
\langle x, y\rangle=\sum_{i=1}^{k} x^{i} y^{i} \text { and }\|x\|^{2}=\langle x, x\rangle \text {. }
$$

If $A$ is a matrix of order $k \times \ell$ let $A^{i j}$ be the entry of $A$. Also, let $A^{t}$ be the transpose of $A$, and let

$$
\|\mathrm{A}\|^{2}=\sum_{i=1}^{\mathrm{k}} \sum_{i=1}^{\ell}\left|\mathrm{A}^{i j}\right|^{2} .
$$


2.2 : Let $M(x), \quad x \in R^{k}$ be an (unknown) twice differentiable function from $\mathrm{R}^{\mathrm{k}} \rightarrow \mathrm{R}^{\mathrm{k}}$. Let $\theta_{\mathrm{n}}(\mathrm{n}=1,2, \ldots)$ be (unknown) vectors in $R^{k}$. The first $\theta_{1}$, being the unique root of the equation $M(x)=0$, Set $M_{l}(x)=M(x)$; for $n=1,2, \ldots$ set $M_{n}(x)=M\left(x-\theta_{n}+\theta_{1}\right)$ so that $\theta_{n}$ is the unique root of $H_{11}(x) \cdot$

2.3 : Let $D(x)$ be the derivative of $M(x), i . e ., D^{i j}=\left(\partial / \partial x_{j}\right) M^{i}(x)$ and assume the following assumptions on D

i) $D\left(\theta_{1}\right)$ is ron singular

ii) For all $\varepsilon>0$

$$
\inf \left\{\left\|D^{t}(x) M(x)\right\|: \varepsilon<\|M(x)\| \leq \varepsilon^{-1}\right\}>0
$$

ii $) \sup \left\{\|D(x)\|: x \in R^{k}\right\}<\infty$.

2.4 : Let $H(x)$ be the Hessian of $\|M(x)\|^{2}$, i.e.

$$
\begin{aligned}
& \mathrm{H}^{\mathrm{i} j}=\left(\partial^{2} / \partial \mathrm{x}^{\mathrm{i}} \partial \mathrm{x}^{j}\|M(\mathrm{x})\|^{2}\right. \text {. and assume that } \\
& \text { EuF }\left\{\|\mathrm{H}(\mathrm{x})\|: x \in \mathrm{R}^{\mathrm{k}}\right\}<\omega .
\end{aligned}
$$

2.5 : Assume that $\theta_{n}$ moves in a such manner that

$$
\theta_{n+1}=g_{n}\left(\theta_{n}\right)+v_{n}
$$

where $g_{n}(x)$ is in general a non-linear measurable function (known) from $\mathrm{R}^{\mathrm{k}} \rightarrow \mathrm{R}^{\mathrm{k}}$ and $\mathrm{v}_{\mathrm{n}}$ is an unknown (random or nonrandom) $k$-vector function independent of $\mathrm{x}$ and

$$
\left\|v_{n}\right\|=O\left(\delta_{n}\right), \sum_{n=1}^{\infty} \delta_{n}<\infty .
$$

2.6 : For $x$ and $y$ in $R^{k}$, we assume that exists a sequence of positive numbers $\left\{\gamma_{n}\right\}$ independent of $x$ and $y$ and let

$$
z_{n}=x-y, \quad \text { and } \quad{ }^{*}{ }_{n}=g_{n}(x)-g_{n}(y)
$$

Then

$$
\left\|M\left(\stackrel{*}{z}_{n}\right)\right\|^{2} \leq \gamma_{n}\|M(z)\|^{2}
$$




$$
\sum_{n=1}^{\infty}\left(\gamma_{n}-1\right)^{+}<\infty .
$$

where $z^{+}$means $(z+|z|) / 2$.

and

$$
\lim _{n \rightarrow \infty} M\left(\stackrel{*}{z}_{n}\right)=M(z) \quad \text { for }\|z\|<\infty
$$

\section{THE PROCEDURE}

The dynamic Robbins-Monro procedure will be described formally by the following assumptions.

3.1 : Let $x_{1}$ be atbitrary; define

$$
\mathrm{x}_{\mathrm{n}+1}=\stackrel{*}{\mathrm{x}}_{\mathrm{n}}-\mathrm{an}^{-1} \mathrm{~F}_{\mathrm{n}}^{\mathrm{t}} \mathrm{Y}_{\mathrm{n}}
$$

where

$\stackrel{*}{\mathrm{X}}_{\mathrm{n}}=\mathrm{G}_{\mathrm{n}}\left(\mathrm{x}_{\mathrm{n}}\right) ; \mathrm{a}>0 ; \mathrm{x}_{\mathrm{n}}$ in $\mathrm{R}^{\mathrm{k}}$ and $\mathrm{F}_{\mathrm{n}}$ is $\mathrm{k} \times \mathrm{k}$ random matrix, is used to estimate $D_{n+1}\left(x_{n}^{*}\right)$ and $y_{n}$ is the obervation with conditional expectation equal to $M_{n+1}\left(x_{n}^{*}\right)$. The ith column of $F_{n}$ is constructed as follows: Let e(i) be the ith column of the $k \times k$ identity matrix. Let $c_{n}>0$ be constant and let $Y_{n}(i, 2)$ and $Y_{n}(i, 1)$ each be the observation with conditional expectation equal to $M_{n+1}\left(\stackrel{*}{x}_{n}+\right.$ $\left.c_{n} e(i)\right)$ and $M_{n+1}\left(\stackrel{x}{n}_{n}-c_{n} e(i)\right)$, respectively. Then, the ith column of $\mathrm{F}_{\mathrm{n}}$ is

$$
\mathrm{F}_{\mathrm{n}}^{i}=\left[\mathrm{Y}_{\mathrm{n}}(i, 2)-\mathrm{Y}_{\mathrm{n}}(i, 1)\right] / 2 \mathrm{c}_{\mathrm{n}}
$$

Let $F_{n}$ be the o-algebra generated by $x_{1}, x_{2}, \ldots, x_{n}$. For any random vector $x$ in $R^{k}$, let $E_{n}(x)$ and $\operatorname{Var}_{n}(x)$ be respectively the conditional mean and the variance of $x$ given $\widetilde{F}_{n}$. 
Given ${ }^{*} n, Y_{n}, D_{n}$ and $v_{n}$ are conditionally independent. Let

$$
\xi_{n}=Y_{n}-M_{n+1}\left(x_{n}\right) ; \quad E_{n}\left(\xi_{n}\right)=0
$$

Let

$$
\begin{aligned}
& \bar{F}_{n}=E_{n}\left(F_{n}\right) \\
& d_{n 1}=F_{n}-\bar{F}_{n}
\end{aligned}
$$

arid assume that

$$
\begin{aligned}
& E_{n}\left(\left\|\xi_{n}\right\|^{2}\right) \leq \delta^{2}<\infty, \\
& E_{n}\left(\left\|d_{n}\right\|^{2}\right) \leq k c_{n}^{-2}
\end{aligned}
$$

and

$$
\left\|\bar{F}_{n}-D_{n+l}\left(x_{n}\right)\right\| \leq k c_{n}
$$

$3.2: c_{n}>0 ; c_{n} \downarrow 0$ and assume thá

$$
\sum_{n=1}^{\infty} n^{-1} c_{n}<\infty, \quad \sum_{n=1}^{\infty} n^{-2} c_{n}^{-2}<\infty \text {, }
$$

\section{THE MAIN REBUET}

Theorem 4.1.: If the assumptions $2.1-3.2$ hold. Then $x_{n}-\theta_{n} \rightarrow 0$. Proof : Using (2.1) and (3.1) we obtain

$$
x_{n+1}-\theta_{n+1}=g_{n}\left(x_{n}\right)-g_{n}\left(\theta_{n}\right)-v_{n}-a^{-1} F_{n}^{t} Y_{n} \text {. }
$$

Let

$$
z_{n+1}=x_{n+1}-\theta_{n+1}, z_{n}^{*}=g_{n}\left(x_{n}\right)-g_{n}\left(\theta_{n}\right) \text { and } \Delta_{n}=a n^{-1} F_{n}^{t} y_{n}
$$

Then (4.1) can be written as

$$
z_{n+1}=\stackrel{*}{z}_{n}-v_{n} \Delta_{n}
$$

and there is $n$ in $(0,1)$ such that

$$
\begin{aligned}
\left\|M\left(z_{n+1}\right)\right\|^{2}= & \left\|M\left(\stackrel{z}{z}_{n}^{*}\right)\right\|^{2}-2\left\langle v_{n}+\Delta_{n}, D^{t}\left(\stackrel{z}{z}_{n}\right) M\left(\stackrel{z}{z}_{n}\right)\right\rangle+\frac{1}{2}\left\langle v_{n}+\Delta_{n},\right. \\
& \left.H\left(\stackrel{z}{z}_{n}^{*}-n\left(v_{n}+\Delta_{n}\right)\right)\left(v_{n}+\Delta_{n}\right)\right\rangle .
\end{aligned}
$$

Using 2.3 and $(3.3)-(3.5)$ we get 


$$
\begin{aligned}
& \left.E_{n}\left(\left\|M_{2}\left(z_{n+1}\right)\right\|^{2}\right)=\left\|M\left(\stackrel{z}{z}_{n}^{*}\right)\right\|^{2}-2 E_{n}\left(\Delta_{n}\right), D^{t}\left({ }^{*}{ }_{n}\right) M\left(z_{n}^{*}\right)\right\rangle-2\left\langle E_{n}\left(V_{n}\right),\right. \\
& \left.D^{t}\left(\stackrel{*}{Z}_{n}\right) M\left(\stackrel{*}{Z}_{n}\right)\right\rangle+\frac{1}{2} E_{n}\left[\left\langleV_{n}+\Delta_{n}, H\left(\stackrel{*}{Z}_{n}-\right.\right.\right. \\
& \left.\left.r\left(v_{n}+\Delta_{n}\right)\right)\left(v_{n}+\Delta_{n}\right)>\right] \\
& \leq\left\|M\left(\stackrel{*}{Z}_{n}\right)\right\|^{2}-2 a n^{-1}\left\|D^{t}\left(\stackrel{*}{Z}_{n}\right) M\left(\stackrel{*}{Z}_{n}^{*}\right)\right\|^{2}-2 a n^{1-}<\left[F_{n}^{-t}-\right. \\
& \left.\left.D^{t}\left(z_{n}\right)\right] M\left(\stackrel{\star}{z}_{n}\right), D^{t}\left(\stackrel{\star}{z}_{n}\right) M\left(\stackrel{\star}{Z}_{n}\right)>+O\left(\left\|v_{n}\right\|\right)\left\|D^{t}\left(\stackrel{*}{z}_{n}\right) M\left(\stackrel{*}{Z}_{n}\right)\right\|\right)+ \\
& 0\left(n^{-2} E_{n}\left(\left\|F_{n}^{t} Y_{n}\right\|^{2}\right)\right)+o\left(\left\|v_{n}\right\|^{2}\right)+o\left(\left\|v_{n}\right\| E_{n}\left\|F_{n}^{t} Y_{n}\right\|\right) \text {. }
\end{aligned}
$$

Hence

$$
E_{n}\left(\left\|M\left(z_{n+1}\right)\right\|^{2}\right)=\| M\left(\left(_{z_{1}}^{*}\right)\left\|^{2}-2 a n^{-1}\right\| D^{t}\left(\stackrel{*}{z}_{n}\right) M\left(\stackrel{*}{z}_{n}\right) \|^{2}+\sum_{i=1}^{5} T_{i}\right.
$$

where $\mathrm{T}_{i} \quad \mathrm{i}=1,2, \ldots, 5$ are the corresponding, terms respectively in (4.3) By (3.8) we have

$$
\begin{aligned}
& \left|T_{1}\right|=0\left(n^{-1} C_{n}\left\|M\left(\stackrel{Z}{Z}_{n}^{\star}\right)\right\|^{2}\right) \\
& \left|T_{2}\right|=0\left(\left\|v_{n}\right\|\left\|D^{+}\left(\sum_{n}^{*}\right) M\left(z_{n_{1}}^{*}\right)\right\|^{2}\right)+\left\|v_{n}\right\|
\end{aligned}
$$

By $(3.3)-(3.8)$ we have

$$
\begin{aligned}
& \left.E_{n}\left(\| F_{2}{ }^{t}{ }_{Y}\right) \|^{2}\right)=\left\|D^{t}\left(\stackrel{*}{Z}_{n}\right) M\left(\stackrel{*}{Z}_{n}\right)\right\|^{2}+M^{t}\left(\stackrel{*}{Z}_{n}\right)\left[F_{n} F_{n}^{-t}-D\left(Z_{n}\right) D^{t}\left(Z_{n}\right)\right] M\left(\stackrel{Z}{Z}_{n}\right) \\
& +E_{n}\left[\left\|d_{n}^{t} \xi_{n}\right\|^{2}\right]+E_{n}\left[\left\|F_{n}^{-t} \xi_{n}\right\|^{2}\right] \\
& \leq\left\|D^{t}\left(z_{n}\right) M\left(z_{n}\right)\right\|^{2}+o\left[\left(c_{n}+c_{n}^{-2}\right)\left\|M\left(z_{n}^{*}\right)\right\|^{2}\right] \\
& +o\left(1+c_{n}+c_{n}^{-2}\right) \text {. }
\end{aligned}
$$

Thus

$$
\begin{aligned}
\left|T_{3}\right|=0\left(n^{-2}\left\|D^{t}\left(\stackrel{*}{z}_{n}\right) M\left(\stackrel{*}{z}_{n}\right)\right\|^{2}\right)+o\left(n^{-2} c_{n}+n^{-2} c_{n}^{-2}\right)\left\|M\left(\stackrel{z}{z}_{n}\right)\right\|^{2} \\
+O\left(n^{-2}+n^{-2} c_{n}+n^{-2} c_{n}^{-2}\right)
\end{aligned}
$$

From which it follows also that

$$
\left|T_{5}\right|=O\left(\left\|v_{n}\right\| E_{n}\left\|\Delta_{n}\right\|\right)=O\left(\left\|v_{n}\right\|+\left\|v_{n}\right\|\left|T_{3}\right|\right)
$$


Substituting $(4.5)-(4.8)$ in (4.5) and using (2.3) we get $E_{n}\left(\left\|z_{n+1}\right\|^{2}\right) \leq\left\|M\left(z_{n}\right)\right\|^{2}\left(1+\mu_{n}\right)-2 a n^{-1}(1+O(1))\left\|D^{t}\left(\stackrel{z}{z}_{n}\right) M\left(\stackrel{z}{z}_{n}\right)\right\|^{2}+\varepsilon_{n}$

where

$$
\mu_{\mathrm{n}}=O\left(\mathrm{n}^{-1} \mathrm{C}_{\mathrm{n}}+\mathrm{n}^{-2} \mathrm{C}_{\mathrm{n}}^{-2}+\gamma_{\mathrm{n}}-1\right)^{+}
$$

and

$$
\varepsilon_{n}=O\left(\left\|v_{n}\right\|+n^{-2} c_{n}^{-2}\right) \ldots \ldots
$$

From (2.2), (2.4) and (3.9) it follows that $\Sigma \mu_{\mathrm{n}}<\infty$ and $\Sigma \varepsilon_{\mathrm{n}}<\infty$. Therefore by Therem 1 of Robbins and siegmund (1971) $\underset{n \rightarrow \infty}{\lim } \mathbf{M}\left(\stackrel{*}{Z}_{n}\right)$ exists and is finite and

$$
\sum_{n=1}^{\infty} n^{-1}\left\|D^{t}\left(\stackrel{z}{z}_{n}\right) M\left(\stackrel{z}{z}_{n}\right)\right\|^{2}<\infty
$$

By 2.2 (ii) and (2.5) $x_{n}-\theta_{n} \longrightarrow 0$, which completes the proof of the theorem.

\section{FEFEREINCES}

[1] Dupac, V. (1965), Adynamic stochastic approximation method. The Anal. Math. Statist. 36, 1695-1702.

[2] Dupac, V. (1966), stochastic aproximation in the presence of the trend. Czechosloak Mathematical Jounal,16, 454-461.

[3] Robbins, H. and Monoro, S. (1951), A stochastic approximation method. Ann. Math. Statist. 22, 400-407.

[4] Robbins, H. and Siegmund, D. (1971), A convergence theorem for non-negative almost super martingles and some applications. In optimizing Methods in statistics (J.S. Rustagi, ed.) 233-257, Academic, New York.

[5] Ruppert, D. (1985), A Newtor-Raphson version of the multivariate Robbins-Monoro procedure. Ann statist. 13, $236-245$.

[6] Sorour, E. (1978), on the convergence of the dynamic stochastic approximation method for stochastic non-linear multidimensional dynamic dystems. Kybernetika, Volume 14, $28-37$.

[7] Sorour, E. (1991), A dynamic Kiefer-Wolfowitz stochastic approximation procedure. Statistics \& Decision 9, 201-211.

[8] Sorour, E. (1993), Dynamic multivariate stochastic approximation using a simultaneous perturbation gradient approximation.(appear).

[9] Uosaki, K. (1974), Some generalization of the dynamic stochastic approximation process. Ann. Statist. 2, 1042-1048. 